THE Astrophysical JoURNAL, 497:713-716, 1998 April 20

(C) 1998. The American Astronomical Society. All rights reserved. Printed in U.S.A.

\title{
NONACCELERATION OF SAGITTARIUS A*: IMPLICATIONS FOR GALACTIC STRUCTURE
}

\author{
ANDREW GOULD ${ }^{1}$ AND SOLANGE V. RAMÍREZ \\ Department of Astronomy, Ohio State University, Columbus, OH 43210; gould@astronomy.ohio-state.edu, \\ solange@astronomy.ohio-state.edu \\ Received 1997 May 27; accepted 1997 November 19
}

\begin{abstract}
We estimate that observations by Backer and collaborators over the past two decades constrain the time derivative of the proper motion of $\mathrm{Sgr} \mathrm{A}^{*}$ to be less than 0.14 mas $\mathrm{yr}^{-2}$. Using this result and a measurement by Eckart \& Genzel of $\sigma \sim 560 \mathrm{~km} \mathrm{~s}^{-1}$ for the velocity dispersion of the star cluster within 0.3 of Sgr A*, we derive the following implications. First, if the nuclear star cluster is dominated by a massive black hole, then either Sgr A* is directly associated with that black hole or it orbits the black hole with a radius $\lesssim 40 \mathrm{AU}$. Under this assumption, and adopting $v_{\mathrm{LSR}}=220 \pm 10 \mathrm{~km} \mathrm{~s}^{-1}$, the Galactocentric distance is therefore $R_{0}=7.5 \pm 0.4 \mathrm{kpc}$. If the star cluster is not assumed to contain a massive black hole, Sgr A* is constrained to move slower than $55 \mathrm{~km} \mathrm{~s}^{-1}(1 \sigma)$ relative to the center of mass of the cluster. This is too weak a limit to provide useful constraints on Galactic structure, but we show that it could be improved by more than one order of magnitude over the next decade. Such an improvement would eliminate the need to make any assumption about a black hole in the derivation of $R_{0}$.

Subject headings: astrometry - Galaxy: center - Galaxy: fundamental parameters open clusters and associations: individual (Sgr A*)
\end{abstract}

\section{INTRODUCTION}

Backer \& Sramek (1987) and Backer (1996) have measured the proper motion of the compact nonthermal radio source Sgr A* and find values that are in reasonable agreement with those expected from the reflex motion of the Sun, assuming a local standard of rest (LSR) rotation speed $v_{\mathrm{LSR}}=220 \mathrm{~km} \mathrm{~s}^{-1}$ and a Galactocentric distance $R_{0}=7.5$ $\mathrm{kpc}$. The $1 \sigma$ error bars $\left(0.17 \mathrm{mas} \mathrm{yr}^{-1}\right)$ correspond to $\sim 6$ $\mathrm{km} \mathrm{s}^{-1}$ at a distance of $7.5 \mathrm{kpc}$.

This agreement has been used as the basis for two related arguments. Backer (1996) reasons that since the source is moving at most very slowly relative to the proper motion of the Galactic center as predicted from "known" Galactic parameters, it must be a black hole of at least $100 M_{\odot}$. On the other hand, Reid (1993) reasons that since the source is very likely to be at rest with respect to the center of mass of the Galaxy, one can use its proper motion to measure $v_{\mathrm{LSR}} / R_{0}$, and so (to the extent that $v_{\mathrm{LSR}}$ is considered known) constrain $R_{0}$. Each argument is important and interesting but clearly both cannot be used together.

Here we show that upper limits on the time derivative of the proper motion of $\mathrm{Sgr} \mathrm{A}^{*}$ constrain its motion relative to the nuclear star cluster at the Galactic center. We explore what conclusions can be drawn from this constraint, both now and in the future.

\section{OBSERVATIONAL DATA}

Comparing the results of Backer \& Sramek (1987),

$\left(\mu_{l}, \mu_{b}\right)=(-5.95 \pm 0.70,+0.43 \pm 0.50){\text { mas } \mathrm{yr}^{-1}}^{-1}$

and Backer (1996),

$\left(\mu_{l}, \mu_{b}\right)=(-6.55 \pm 0.17,-0.48 \pm 0.12){\text { mas } \mathrm{yr}^{-1}}^{-1}$

\footnotetext{
${ }^{1}$ Alfred P. Sloan Foundation Fellow.
}

it is clear that the proper motion of Sgr A* did not change much over a decade. It is difficult to give a precise upper limit to the time derivative of the proper motion because the underlying data have not been published. For purposes of this paper, we estimate the upper limit by combining equation (2.1) and (2.2) with the data points shown in Figure 1 of Backer \& Sramek (1987) and find

$$
\left[\left(\frac{d^{2} l}{d t^{2}}\right)^{2}+\left(\frac{d^{2} b}{d t^{2}}\right)^{2}\right]^{1 / 2}<0.14{\operatorname{mas~} \mathrm{yr}^{-2}}^{-2}
$$

at the $1 \sigma$ level, corresponding to a limit on the physical transverse acceleration $a_{\perp}$ of

$$
a_{\perp}<a_{\max }=5.3 \mathrm{~km} \mathrm{~s}^{-1} \mathrm{yr}^{-1} \sim 0.028 a_{\oplus},
$$

where we have for simplicity of exposition adopted $R_{0}=$ $8 \mathrm{kpc}$, and where $a_{\oplus}$ is the acceleration of the Earth about the Sun.

From the work of Menten et al. (1997) the position on infrared images corresponding to the radio position of Sgr $A^{*}$ is now known to an accuracy of 0"03. Eckart \& Genzel (1997) have measured the proper motions of stars in the infrared within $2^{\prime \prime}(\sim 0.1 \mathrm{pc})$ of this position. In general, they find that the velocity dispersion rises toward the center in a way that is consistent with a central black hole with mass $M_{*}=2.45 \times 10^{6} M_{\odot}$. In particular, for the measurement at the innermost point, they find a one-dimensional velocity dispersion $\sigma \sim 560 \mathrm{~km} \mathrm{~s}^{-1}$.

For present purposes, it is important to estimate the characteristic radius to which this measurement applies. We do so in three ways. First, the angular separation between Sgr $A^{*}$ and the centroid of the 11 stars used to make the measurement is $\theta_{* \text {, cent }}=0^{\prime \prime} 22$. Second, the harmonic mean separation between Sgr $\mathrm{A}^{*}$ and the 11 stars is $\theta_{* \text {,harm }}=$ $0^{\prime \prime} 21$. Third, by the virial theorem, the characteristic radius corresponding to a mass $M_{*}$ and dispersion $\sigma$ is $G M_{*} / 3 \sigma^{2}$. The first two quantities are projected onto the plane of the 
sky, while the third is the radius of a three-dimensional sphere. In order to compare them, we therefore multiply the first two by $(3 / 2)^{1 / 2} R_{0}$ and find

$$
\begin{gathered}
\left(\frac{3}{2}\right)^{1 / 2} R_{0} \theta_{* \text {, ent }}=2200 \mathrm{AU} \\
\left(\frac{3}{2}\right)^{1 / 2} R_{0} \theta_{* \text {, harm }}=2100 \mathrm{AU}, \quad \frac{\mathrm{GM}_{*}}{3 \sigma^{2}}=2300 \mathrm{AU} .
\end{gathered}
$$

That is, all methods lead to the same estimate of the characteristic radius of the stellar cluster. To be conservative, we adopt a somewhat larger radius, $r_{*}=3000 \mathrm{AU}$. The star cluster is then characterized by a mass $M_{*}$ contained within a radius $r_{*}$ :

$$
M_{*}=2.45 \times 10^{6} M_{\odot}, \quad r_{*}=3000 \mathrm{AU} .
$$

Hence, the magnitude of the acceleration at the boundary of this region is

$$
a\left(r_{*}\right)=\frac{M_{*} / M_{\odot}}{\left(r_{*} / \mathrm{AU}\right)^{2}} a_{\oplus} \sim 0.27 a_{\oplus} .
$$

In accordance with equation (2.5), we assume that Sgr A* lies within the radius, $r_{*}$.

Eckart \& Genzel (1997) describe the measurement at this last point as still "preliminary." However, at the $23 \mathrm{~d}$ General Assembly of the IAU, two other groups reported similar measurements, and R. Genzel (1997, private communication) now regards the Eckart \& Genzel (1997) result as "confirmed." The weakest part of the argument presented in this paper is therefore the estimate of $a_{\max }$ given in equation (2.4). As we discussed above, this is based on our reconstruction of unpublished data. It therefore could be subject to significant revision when the data are published. D. Backer (1997, private communication) plans to publish the data on which our estimate is based along with a thorough error analysis. When a new determination of $a_{\max }$ is made, the results presented here can simply be rescaled.

Equations (2.4) and (2.7) reveal the basic result that we will exploit in this paper: the ratio, $\epsilon$, of the upper limit for the transverse acceleration of $\mathrm{Sgr} \mathrm{A}^{*}$ to the characteristic acceleration of the system in which it is embedded is small,

$$
\epsilon \equiv \frac{a_{\max }}{a\left(r_{*}\right)} \sim 0.10
$$

To understand the implications of this result, we consider two limiting cases: first where the mass $M_{*}$ is dominated by a single point mass (a black hole), and second where $M_{*}$ is distributed uniformly throughout the region inside $r_{*}$. We show that in the first case, Sgr A* either is directly associated with the black hole or it is orbiting it closely. In the second, the constraints on the motion Sgr A* relative to the nuclear star cluster are substantially weaker.

\section{KEPLER POTENTIAL}

Suppose that the region within $r_{*}$ is dominated by a massive black hole. Then there are two possibilities: either Sgr A* is directly associated with the black hole, or it is orbiting in the potential of the black hole. If the first is true, our case is already proved, so we restrict consideration to the second.
We designate the position of Sgr $\mathrm{A}^{*}$ relative to the black hole by $(r, \theta, \phi)$ were $\theta$ is the angle Sun-black hole-Sgr A*. Then,

$$
\sin \theta=\frac{a_{\perp}}{a(r)}<\frac{a_{\max }}{a(r)}=\epsilon\left(\frac{r}{r_{*}}\right)^{2} .
$$

The prior probability for such a fortuitous geometry at any given instant (given that $r<r_{*}$ ) is small, less than $\left(\frac{3}{10}\right) \epsilon^{2} \sim$ $3 \times 10^{-3}$ for a monotonically decreasing density profile. Even if Sgr A* happened to lie sufficiently close to the line of sight to the black hole at the beginning of the observations, it would move out of this zone within the $T \sim 10 \mathrm{yr}$ of observations unless it were on a highly radial orbit, with its tranverse speed $v_{\perp}$ constrained by $v_{\perp}<v_{\max }=$ $\epsilon r^{3} / r_{*}^{2} T \sim 140 \mathrm{~km} \mathrm{~s}^{-1}\left(r / r_{*}\right)^{3}$. This further reduces the prior probability by a factor $\left(v_{\max } / \sigma\right)^{2} / 2$ to a net probability of $\lesssim 10^{-4}$. That is, this scenario is essentially ruled out.

Hence, if there is a large black hole in the center of the nuclear star cluster, then Sgr A* must be directly associated with it. The one potential loophole is that Sgr $\mathrm{A}^{*}$ might be physically associated with the black hole and orbit it with a period much shorter than the frequency of observations, $\sim(450 \text { day })^{-1} \quad$ (Backer \& Sramek 1987). The physical association would evade the above probability argument, and the short period would imply that Sgr A* would orbit many times between observations and therefore would not show any secular acceleration. However, for an orbital radius $r \lesssim 150 \mathrm{AU}$ (corresponding to a period less than 450 days), the typical displacement between observations would be $\sim r / R_{0} \sim 20$ mas $(r / 150 \mathrm{AU})$. The actual displacements from uniform motion are $\lesssim 5$ mas (see Fig. 1 from Backer \& Sramek 1987), implying that Sgr A* has an orbital radius $r \lesssim 40 \mathrm{AU}$, and therefore a speed $v \gtrsim 7000 \mathrm{~km} \mathrm{~s}^{-1}$. However, even if Sgr A* were in such an orbit (as opposed to being directly associated with the black hole) its observed proper motion would still be equal to the proper motion of the black hole. The $\sim 40 \mathrm{AU}$ orbit (compared to the $\gtrsim 700$ AU that Sgr A* has been observed to move relative to the Sun over the lifetime of the observations) would "explain" the observed scatter, which presently is attributed to unknown systematic errors. Regardless of the assumed cause, however, the scatter is already taken into account in the analysis.

\section{HARMONIC OSCILLATOR POTENTIAL}

Next, we suppose that the mass within $r_{*}$ is not concentrated at a point, but rather is distributed throughout the region, perhaps in the form of stars or possibly other objects. Most likely, the density profile would be monotonically decreasing, but for simplicity and to focus on an extreme case, we consider a uniform distribution. We note that there are many potential problems for a star cluster of this density because of the shortness of the relaxation time. However, the mass need not be in the form of stars but could be in much lighter particles such as weakly interacting massive particles (WIMPs). Alternatively, the problems associated with dense star clusters might be avoided by some effect that has so far escaped recognition. Since our purpose is to develop completely general arguments, we do not make any assumption about the nature of the material within $r_{*}$, other than that it has total mass $M_{*}$.

A uniform distribution gives rise to a harmonic oscillator potential, so $a(r)=\left(r / r_{*}\right) a\left(r_{*}\right)$. Thus, the analog of equation 
(3.1) is

$$
\rho=r \sin \theta=\frac{a_{\perp}}{a(r)} r<\frac{a_{\max }}{a\left(r_{*}\right)} r_{*}=\epsilon r_{*},
$$

where $\rho$ is the projected separation of Sgr A* from the center of the cluster. The prior probability for this is $(3 /$ 2) $\epsilon^{2} \sim 1.5 \times 10^{-2}$. As in the case of the Kepler potential, Sgr A* would have to be on a nearly radial orbit. Including both effects, the prior probability is $\left(\frac{3}{4}\right) \epsilon^{4}\left(r_{*} / \sigma T\right)^{2} \sim$ $5 \times 10^{-4}$. Again, the scenario is ruled out.

The one exception to this argument would be if Sgr A* were gravitationally confined to be near the center. Then it would not be at a random position in the cluster, and the previous probability argument would fail. In order to be sufficiently confined to satisfy equation (4.1), $\rho<\epsilon r_{*}$, its characteristic speed would be constrained by

$$
v \lesssim \epsilon \sigma \sim 55 \mathrm{~km} \mathrm{~s}^{-1} \text {. }
$$

If the density profile fell monotonically (giving rise to a potential intermediate between Kepler and harmonicoscillator) the arguments presented in this section would still hold but with greater force: the fraction of phase space satisfying the constraint (2.4) would be even smaller than $5 \times 10^{-4}$, and the maximum of velocity of an object gravitationally confined to a region that did satisfy the constraint would be even less than $55 \mathrm{~km} \mathrm{~s}^{-1}$.

We note in passing that by equipartition, the minimum mass of Sgr A* required for it to be gravitationally confined as described above is $M_{\mathrm{Sgr}^{*}}>\epsilon^{-2} M \sim 100 M$, where $M$ is the characteristic mass of the objects in the cluster.

\section{IMPLICATIONS FOR GALACTIC STRUCTURE}

Unfortunately, at the present time no hard and fast conclusions can be drawn from the lack of observed acceleration of Sgr A* because the upper limit for this acceleration is based upon unpublished and reconstructed data. Nevertheless, this upper limit could be confirmed or even strengthened within a few years. We therefore begin by assuming that it will be confirmed and investigate the consequences.

If one is convinced that the high dispersion of the central star cluster implies the presence of massive black hole, then the results of $\S 3 \mathrm{imply}$ that either Sgr $\mathrm{A}^{*}$ is directly associated with that black hole or it orbits the black hole with a radius $r \lesssim 40$ AU. In either case, the observed proper motion of $\mathrm{Sgr} \mathrm{A}^{*}$ is due entirely (within measurement errors) to the reflex motion of the Sun relative to the Galactic center, so one can apply the approach of Reid (1993) to constrain $R_{0}$. To be specific, we adopt an estimate with $1 \sigma$ error of $v_{\mathrm{LSR}}=220 \pm 10 \mathrm{~km} \mathrm{~s}^{-1}$. We note in passing that the small size of this error bar rests critically on the assumption that the rotation curve of the Galaxy, like that of similar external galaxies, is flat. With this assumption, measurement of the redshifts of tangent points interior to the Sun lead to an estimate very close to $v_{\mathrm{LSR}} \sim 220 \mathrm{~km} \mathrm{~s}^{-1}$ (Brand \& Blitz 1992). If this assumption is dropped, the error estimate increases by several fold. We assume that the Sun is moving at $12 \mathrm{~km} \mathrm{~s}^{-1}$ relative to the LSR, or $232 \pm 10$ $\mathrm{km} \mathrm{s}^{-1}$ relative to the Galactic frame. We make use of Backer's (1996) measurement and $1 \sigma$ error, $\mu_{l}=-6.55$ $\pm 0.17 \mathrm{mas} \mathrm{yr}^{-1}$ and find

$$
R_{0}=7.5 \pm 0.4 \mathrm{kpc} \quad \text { (provisional) }
$$

If one drops the assumption that the Galactic center harbors a massive black hole, then by equation (4.2) Sgr A* is still constrained to be moving $v \lesssim 55 \mathrm{~km} \mathrm{~s}^{-1}$ relative to the Galactic center. Unfortunately, this constraint is too weak to yield useful information about $R_{0}$ because the resulting error, $\pm 1.8 \mathrm{kpc}$, is not competitive with other measurements. However, the limit on $v$ is proportional to $a_{\max } r_{*}^{2}$, and it could be improved substantially as we discuss in $\S 6$.

It is also possible to use the proper motion of $\mathrm{Sgr} \mathrm{A}^{*}$ to probe an entirely different question: whether the nuclear star cluster at the Galactic center is at rest with respect to the center of mass of the Galaxy. At a distance of $7.5 \mathrm{kpc}$, Backer's (1996) proper-motion measurement in the $b$ direc-

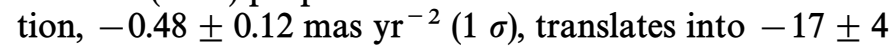
$\mathrm{km} \mathrm{s}^{-1}$. The Sun's motion relative to the LSR is $7 \mathrm{~km} \mathrm{~s}^{-1}$ and is extremely well measured, with an uncertainty of $\ll 1$ $\mathrm{km} \mathrm{s}^{-1}$ (Mihalas \& Binney 1981). Hence there is a net motion of $-10 \pm 4 \mathrm{~km} \mathrm{~s}^{-1}$ that remains unexplained. At the present time, it is not possible to draw any conclusion about this residual for three reasons. First, the effect itself is detected at only the $2.5 \sigma$ level and so could be just a statistical fluctuation. Second, the LSR may be moving relative to the Galactic frame because of a warp in the disk or some other effect. It is possible to directly test this hypothesis (see below). Third, the observed deviation is completely consistent with the constraint (4.2) on the motion of Sgr A* relative to the cluster. This limit is directly proportional to $\epsilon$ and so to $a_{\max }$ (see eq. [2.8]). As discussed in § 6, this limit could be dramatically improved by future observations, which would also improve the statistical error on the proper motion in the $b$ direction. If the discrepancy persisted, there would remain two potential causes, motion of the LSR and motion of the central star cluster (e.g., Miller \& Smith 1992), both of which are interesting possibilities.

The motion of the LSR can be investigated by finding the mean motion of the Sun relative to stars in the Galactic halo. Popowski \& Gould (1997) find that the vertical component of this motion is $7 \pm 6 \mathrm{~km} \mathrm{~s}^{-1}$, that is, $0 \pm 6 \mathrm{~km} \mathrm{~s}^{-1}$ relative to the LSR. Their analysis is based on combining two of the three available samples of halo stars, 106 metalpoor RR Lyrae stars from Layden et al. (1996), and 724 non-kinematically selected metal-poor stars from Beers \& Sommer-Larson (1995). There is a third available sample, 1352 high proper-motion stars analyzed by Casertano, Ratnatunga, \& Bahcall (1990). However, these authors did not fit for the vertical motion of the halo population. This is truly unfortunate because at this point it would not be at all easy to reimplement the beautiful technique they devised to remove even unrecognized selection biases in their samples. If their samples were reanalyzed, however, we estimate that the error in the $z$ motion would be $\sim 5 \mathrm{~km} \mathrm{~s}^{-1}$. Thus, by combining the Casertano et al. (1990) sample with the results of Popowski \& Gould (1997), one could reduce the uncertainty to $\sim 4 \mathrm{~km} \mathrm{~s}^{-1}$. Hence, if the discrepancy persists between the $z$ motion of the LSR and that of Sgr A*, it should be possible to decide which of them is actually moving relative to the Galactic center of mass.

\section{FUTURE IMPROVEMENTS}

The primary obstacle to using the nonacceleration of Sgr $A^{*}$ to constrain Galactic structure is the relatively weak limit on $\epsilon \propto a_{\max } r_{*}^{2}$. In particular, a factor $\sim 10$ improvement on this parameter would be required to bring the limit 
on motion of Sgr A* relative to the nuclear star cluster (eq. [4.2]) well below the uncertainty in $v_{\text {LSR }}$. If this were achieved, the measurement of $R_{0}$ given by equation (5.1) would be completely independent of any assumption about the nature of Sgr A*. Clearly, improvements in $\epsilon$ can only be made by reducing $a_{\max }$ or $r_{*}$.

The limit $a_{\max }$ can probably be improved simply by fitting existing data to a second order polynomial. In any event, continued observations at a uniform rate and with uniform quality would yield a rapid improvement in the precision of this quantity, $\propto T^{-5 / 2}$, where $T$ is the total duration of the observations. In addition, the results obtained to date have used the VLA. A new program of observations using the longer baseline of VLBI has been initiated by M. Reid (1997, private communication), and this should lead to substantial improvements both in the proper motion and its time derivative.

Reducing $r_{*}$ appears more daunting at first sight. The 11 stars measured by Eckart \& Genzel (1997) at their innermost point is close to the minimum required for a good statistical determination of the dispersion. Yet, these observations were already confusion-limited despite the near-diffraction-limited observations on a $4 \mathrm{~m}$ class telescope. Some improvement is clearly possible (and has already been achieved but not yet published) by getting to the diffraction limit on the $10 \mathrm{~m}$ Keck telescope. And one might imagine that future infrared interferometers will do better yet. However, there is another route to reducing $r_{*}$ : direct measurement of the orbital acceleration of the stars already observed by Eckart \& Genzel (1997). The typical orbital period of stars with dispersion $\sigma$ in a potential dominated by a mass $M_{*}$ is

$$
P \simeq \frac{M_{*} / M_{\odot}}{3^{3 / 2}\left(\sigma / v_{\oplus}\right)^{3}} \mathrm{yr}=72 \mathrm{yr}
$$

where $v_{\oplus}=30 \mathrm{~km} \mathrm{~s}^{-1}$ is the speed of the Earth. A significant change in velocity (which makes possible a measurement of the acceleration) occurs after $P / 2 \pi \sim 12 \mathrm{yr}$. The observed acceleration vector (projected onto the plane of the sky) of each star gives the direction to the center of the potential, so even two such stars would allow one to triangulate this position and measure its projected separation from Sgr A*. More stars would allow a check. Moreover, the magnitude of a star's acceleration would give the mass interior to the star, in principle permitting one to push $r_{*}$ in to the position of the closest star $\left(\sim 00^{\prime \prime} 09\right)$, which by itself would yield a $\sim 12$-fold reduction of $\epsilon$. Actually, the unknown inclination of the orbit would create an ambiguity in this determination. The ambiguity could be resolved either statistically (by measuring accelerations for several stars), or by obtaining a radial velocity, or by observing the star long enough to solve for its orbit (as with visual binaries). In brief, it is reasonable to expect that $\epsilon$ can be reduced by one order of magnitude or more over the next decade.

There is one other, less severe, loophole that can also be addressed by future observations. In $\S 3$, we showed that if Sgr A* were orbiting a massive black hole, then the orbit must be $r \lesssim 40$ AU. Clearly, this bound can be improved in inverse proportion to the accuracy of the astrometric measurements, but can the loophole be eliminated entirely, even for very small orbits, $r<10 \mathrm{AU}$ ? It is possible to test directly for such small orbits by looking for time variability of the flux due to the Doppler effect. The fractional amplitude of the flux oscillations would be $f \sim(1+p) v \sin i / c$, where $p \sim 0.33$ is the slope of power law $\left(S_{v} \propto v^{p}\right.$; Mezger 1996), $i$ is the orbital inclination, and $v$ is the orbital velocity. Thus $f \sim 0.07(r / 10 \mathrm{AU})^{-1 / 2} \sin i$ with period $\sim 7$ days $(r / 10 \mathrm{AU})^{3 / 2}$.

We conclude that the nonacceleration of Sgr A* already places useful constraints on Galactic structure, and that these constraints can be made substantially tighter over the next decade.

We thank G. Newsom for a careful reading of the manuscript. The paper profited from an exceptionally helpful referee report from D. Backer. This work was supported in part by grant AST 94-20746 from the NSF, and in part by grant NAG 5-3111 from NASA.

\section{REFERENCES}

Backer, D. C. 1996, in IAU Symp. 169, Unsolved Problems of the Milky

Menten, K. M., Reid, M. J., Eckart, A., \& Genzel, R. 1997, ApJ, 475, L111 Way, ed. L. Blitz \& P. Teuben (Dordrecht: Kluwer), 193

Backer, D. C., \& Sramek, R. A. 1987, in AIP Conf. Proc. 155, ed. D. L. Backer (New York: AIP), 163

Beers, T. C., \& Sommer-Larsen, J. 1995, ApJS, 96, 175

Brand, J., \& Blitz, L. 1992, A\&A, 275, 67

Casertano, S., Ratnatunga, K. U., \& Bahcall, J. N. 1990, ApJ, 357, 435

Eckart, A., \& Genzel, R. 1997, MNRAS, 284, 576

Layden, A. C., Hanson, R. B., Hawley, S. L., Klemola, A. R., \& Hanley, Mezger, P. G. 1996, in ÄSP Conf. Ser. 102, The Galactic Center, ed. R. Gredel (San Francisco: ASP), 380

Mihalas, D., \& Binney, J. 1981, Galactic Astronomy (San Francisco: Freeman)

Miller, R. H., \& Smith, B. F. 1992, ApJ, 393, 508

Popowski, P., \& Gould, A. 1997, ApJ, submitted

Reid, M. J. 1993, ARA\&A, 31, 345

C. J. 1996, AJ, 112, 2110 